\title{
An approach for the modeling of interface-body coupled nonlocal damage
}

\author{
S. Marfia, E. Sacco, J. Toti \\ University of Cassino, DiMSAT, Italy; sacco@unicas.it
}

RIASSUNTO. I materiali composite fibrorinforzati (FRP) possono essere utilizzati con successo per il rinforzo di costruzioni in conglomerato cementizio ed in muratura. Uno dei maggiori problemi nell'uso degli FRP, che spesso ne limita l'impiego, è la possibile decoesione della lamina in FRP dalla struttura esistente realizzata in materiale coesivo (calcestruzzo o muratura). Il presente lavoro affronta la problematica della modellazione della decoesione di lamine o tessuti FRP dal supporto, tenendo conto del possibile accoppiamento tra il degrado del materiale di supporto (calcestruzzo o muratura) ed il danneggiamento dell'interfaccia FRP-supporto in materiale coesivo. In particolare, per il materiale coesivo di supporto è proposto un modello di danno non locale di tipo integrale, governato dalla media pesata nello spazio di una deformazione equivalente, capace di evitare $\mathrm{i}$ problemi di localizzazione della deformazione e del danno e la forte dipendenza dalla discretizzazione nello schema agli elementi finiti. Relativamente all'interfaccia FRP-supporto, si utilizza un modello di danno locale governato dal valore dello spostamento relativo, tenendo conto della decoesione per modo I, modo II e modo misto. Il modello tiene in conto dell'accoppiamento tra il danno del supporto ed il danno di interfaccia. Alcune applicazioni numeriche sono presentate.

ABSTRACT. Fiber Reinforced Plastic (FRP) can be used for strengthening concrete or masonry constructions. One of the main problem in the use of FRP is the possible detachment of the reinforcement from the support material. This paper deals with the modeling of the FRP-concrete or masonry damage interface, accounting for the coupling occurring between the degradation of the cohesive material and the FRP detachment. To this end, a damage model is considered for the quasi-brittle material. In order to prevent strain localization and strong mesh sensitivity of the solution, an integral-type of nonlocal model based on the weighted spatial averaging of a strain-like quantity is developed. Regarding the interface, the damage is governed by the relative displacement occurring at bond. A suitable interface model which accounts for the mode I, mode II and mixed mode of damage is developed. The coupling between the body damage and the interface damage is performed computing the body damage on the bond surface. Numerical examples are presented.

KEYWORDS. Interface damage; Body damage; Coupled model; FRP strengthening.

\section{INTRODUCTION}

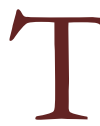

he use of Fiber Reinforced Plastic (FRP) materials for the strengthening of existing concrete and masonry elements is growing and, actually, many structures have been yet reinforced adopting FRP; moreover several experimental and modeling scientific works have been published in the last decade [1-8]. The use of FRP materials applied on the external surface of concrete or masonry structures has aroused new modeling problems. One of the main problem in the use of FRP is the so-called delamination phenomenon, which consists in the sudden decohesion of the FRP reinforcement from the concrete or masonry element, see for instance ref. [9]. In order to model the delamination 
phenomenon and to predict the possible decohesion of the FRP from the support material, special interface models are often adopted. The response of these special elements is mainly based on the damage and/or on plasticity models.

On the other hand, the concrete as well as the masonry are quasi-brittle materials, whose mechanical response is characterized by damage with softening, which is due to the development of micro-cracks. Thus, two damage effects could be present in the quasi-brittle reinforced structural elements: the body damage, which develops inside the domain of the continuous body, and the interface damage, which occurs at the FRP - concrete or masonry interface. It has to be remarked that experimental evidences demonstrate that the detachment of the FRP from the support material occurs with the peeling of a thin layer from the external surface of the quasi-brittle material; this collapse behavior is due to the fact that the strength of the glue used to fix the FRP to the support is greater than the strength of the concrete or masonry support. From this observation, it can be deduced that the body damage and the interface damage cannot evolve independently one from the other, in other words, they are coupled. In particular, the interface damage has to depend on the distribution of the body damage. In the knowledge of the authors, the first paper in which a coupled body-interface damage model has been developed is due to Freddi and Frémond [1].

In the present paper a damage model is proposed for the quasi-brittle material. The damage evolution is governed by an equivalent strain variable. In order to prevent strain localization and strong mesh sensitivity of the solution, typical of cohesive heterogeneous materials subjected to damage and softening, an integral-type of nonlocal model based on the weighted spatial averaging of a strain-like quantity is developed. Regarding the interface, the damage is governed by the relative displacement occurring at bond. A suitable interface model which accounts for the mode I, mode II and mixed mode of damage is developed. The coupling between the body damage and the interface damage is performed ensuring that the interface damage is not lower than the body damage evaluated on the bond surface.

An initial simple application is performed in order to assess the performances of the proposed model in reproducing the mechanical behavior of quasi-brittle material strengthened with external FRP materials.

\section{POSITION OF THE PROBLEM}

A mechanical system made of two bodies $\Omega_{1}$ and $\Omega_{2}$, schematically illustrated in Fig. 1, is considered. The two bodies, subjected to body forces, surface forces and prescribed displacements, are connected by an adhesive interface $\mathfrak{I}=\partial \Omega_{1} \cap \partial \Omega_{2}$, which is assumed to have zero thickness. The problem is developed in the framework of small strain and displacement theory. The displacement fields of the two joined bodies are denoted as $\mathbf{u}^{1}$ and $\mathbf{u}^{2}$, while the relative displacement at the typical point $\mathbf{x}^{\mathfrak{I}}$ on the interface $\mathfrak{I}$ is $\mathbf{s}\left(\mathbf{x}^{\mathfrak{I}}\right)=\mathbf{u}^{1}\left(\mathbf{x}^{\mathfrak{I}}\right)-\mathbf{u}^{2}\left(\mathbf{x}^{\mathfrak{I}}\right)$. A local coordinate system on the interface $\left(x_{T}, x_{N}\right)$ is introduced.

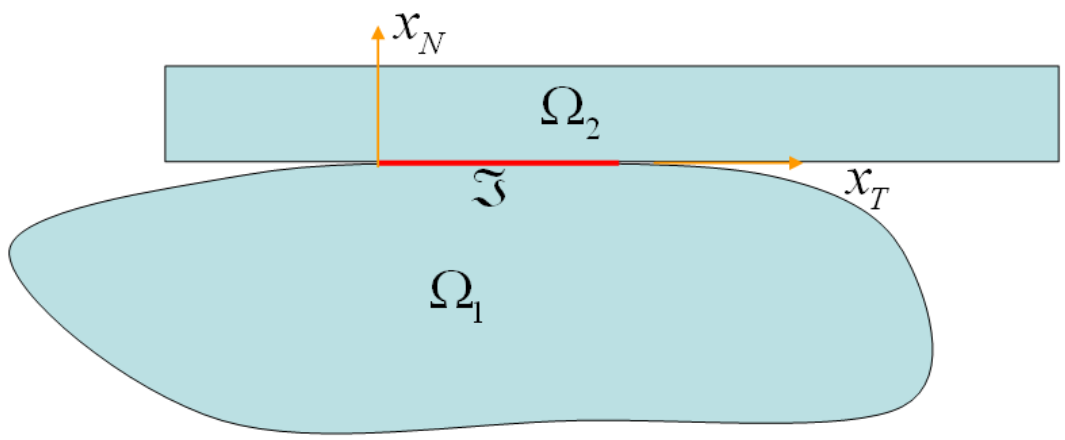

Figure 1: Mechanical system of two bodies in adhesion.

It is assumed that the body $\Omega_{1}$ and the interface $\mathfrak{I}$ can develop damage, while the body $\Omega_{2}$ is assumed to behave as a linear elastic material. Thus, two damage variables are introduced, $D_{\Omega}$ and $D_{\mathfrak{I}}$, associated to the degradation state of the body $\Omega_{1}$ and of the interface $\mathfrak{I}$, respectively.

In many physical problems, the interface failure is not due to the damage of the adhesive material joining the two bodies $\Omega_{1}$ and $\Omega_{2}$, but it occurs because of the properties degradation of a thin layer of the body $\Omega_{1}$; in fact, the decohesion is 
often characterized by the peeling of a thin layer from the external surface of the body $\Omega_{1}$. This observation leads to the consequence that the interface damage depends on the degradation state of the material constituting $\Omega_{1}$. Thus, a coupling between the interface and body damage variables, $D_{\mathfrak{\Im}}$ and $D_{\Omega}$, respectively, arises.

On the other hand, the independent evolution of the two damage variables can lead to physically unacceptable results. The mechanical system illustrated in Fig. 2, representing the scheme of a possible decohesion test, is considered. A damage evolutive model is adopted for the material constituting the body $\Omega_{1}$; regarding the interface, a mode II damage model is assumed. In particular, limiting the analysis to the tangential effect, i.e. neglecting the normal stress, the interface constitutive relationship is written in the form:

$$
\tau_{T}=\left(1-D_{\mathfrak{I}}\right) K_{T} s_{T}
$$

The damage parameter is function of the history of relative displacement as follows:

$$
D_{\mathfrak{J}}=\max _{\text {history }}\left\{\min \left\{1, \tilde{D}_{\mathfrak{J}}\right\}\right\}
$$

where the parameter $\tilde{D}_{\mathfrak{J}}$ is expressed by the relationship :

$$
\tilde{D}_{\mathfrak{\Im}}=\frac{s_{T}^{f}}{s_{T}^{f}-s_{T}^{0}}\left(\frac{\left|s_{T}\right|-s_{T}^{0}}{\left|s_{T}\right|}\right)
$$

with $s_{T}^{0}=\tau_{T}^{0} / K_{T}$ the first cracking relative displacement and $s_{T}^{f}=2 G_{c T} / \tau_{T}^{0}$ the full damage relative displacement, being $\tau_{T}^{0}$ the peak stress on the first cracking relative displacement and $G_{c T}$ the specific fracture energy in mode II. The properties of the materials constituting the considered scheme are the following:

$$
\begin{array}{rlrl}
E_{1} & =16000 \mathrm{MPa} & & v_{1}=0.2 \\
E_{2} & =230000 \mathrm{MPa} & v_{2}=0.0 & \\
K_{T}=1500 \mathrm{~N} / \mathrm{mm}^{3} & \tau_{T}^{0}=3 \mathrm{MPa} \quad G_{c T}=0.3 \mathrm{~N} / \mathrm{mm}^{3}
\end{array}
$$

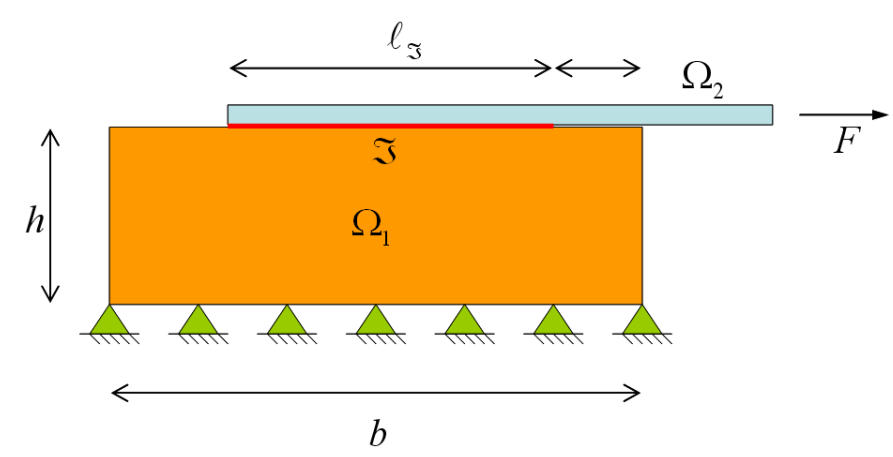

Figure 2: Scheme for decohesion test.

Finite element analyses are performed in order to evaluate the maximum value of the carried force $F_{\max }$ before the decohesion of the body $\Omega_{2}$ from the body $\Omega_{1}$, for different lengths of adhesion. Computations are developed considering the damage state of the body $\Omega_{1}$ assigned and constant during the whole analysis, while the interface damage evolves during the loading history. Thus, the body and interface damages are uncoupled. In Fig. 3, the value of $F_{\max }$ is plotted versus the adhesion length. Note that $D_{I}$ denotes the initial value of the interface damage. It can be remarked that increasing the adhesion length, initially the value of $F_{\max }$ grows, till the optimal adhesion length $\ell_{e}$ is reached, after which $F_{\max }$ remains constant. Moreover, it can be emphasized that for higher values of the damage state of the body $\Omega_{1}$ 
the optimal adhesion length increases and also the maximum value of $F_{\max }$ increases. While the first results is absolutely expected, the second one appears physically unacceptable, as it implies that more the support material is damaged greater values of the forces can be transmitted from $\Omega_{2}$ to $\Omega_{1}$. This strange effect is due to the uncoupled damage evolution of the body and of the interface.

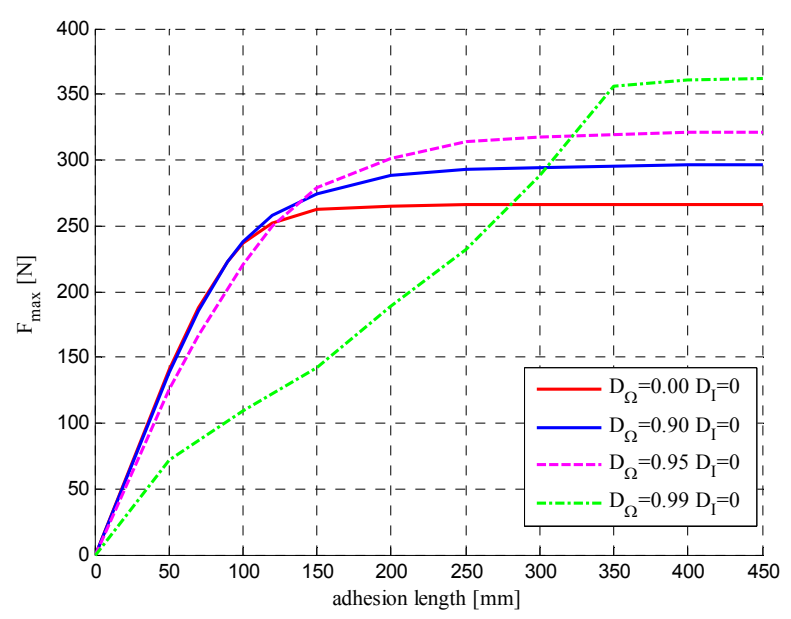

Figure 3: Decohesion force $F_{\text {max }}$ versus adhesion length, uncoupled damage model.

\section{THE FREDDI-FREMOND MODEL}

he Freddi-Fremond (FF) model is based on the assumption of the following forms for the free energy and the pseudo-potential of dissipation of the domain $\Omega_{1}$ :

$$
\begin{aligned}
& \Psi_{\Omega}=\frac{1}{2}\left(1-D_{\Omega}\right)\left[\lambda(\operatorname{tr} \boldsymbol{\varepsilon})^{2}+2 \mu \boldsymbol{\varepsilon} \bullet \boldsymbol{\varepsilon}\right]+w D_{\Omega}+\frac{1}{2} k\left(\nabla D_{\Omega}\right)^{2}+I\left(D_{\Omega}\right) \\
& \Phi_{\Omega}=\frac{1}{2} c\left(\dot{D}_{\Omega}\right)^{2}+I_{+}\left(\dot{D}_{\Omega}\right)
\end{aligned}
$$

where $\boldsymbol{\varepsilon}$ is the strain tensor, $\lambda$ and $\mu$ are the Lamé parameters, $w$ is the initial threshold energy, $k$ is a parameter measuring the nonlocal effect, $I$ and $I_{+}$are the indicator functions of the sets $[0,1]$ and $[0,+\infty]$, respectively, $c$ is the viscosity parameter of damage, while the dot symbol $\bullet$ denotes the scalar product between two tensors, the superposed dot on the damage variable indicates the derivative with respect to the time. The free energy and the pseudo-potential of dissipation of the interface $\mathfrak{I}$ are:

$$
\begin{aligned}
\Psi_{\mathfrak{I}}= & w_{\mathfrak{I}} D_{\mathfrak{I}}+\frac{1}{2} k_{\mathfrak{I}}\left(\nabla D_{\mathfrak{I}}\right)^{2}+I\left(D_{\mathfrak{I}}\right)+I_{-}\left(\left(\mathbf{u}_{2}-\mathbf{u}_{1}\right) \bullet \mathbf{N}\right) \\
& +\frac{1}{2}\left(1-D_{\mathfrak{I}}\right) \hat{k}_{\mathfrak{I}}\left\|\mathbf{u}_{2}-\mathbf{u}_{1}\right\|^{2}+\frac{1}{2} k_{\mathfrak{I}, \Omega}\left(D_{\mathfrak{I}}-D_{\Omega}\right)^{2} \\
\Phi_{\mathfrak{I}} & =\frac{1}{2} c_{\mathfrak{I}}\left(\dot{D}_{\mathfrak{I}}\right)^{2}+I_{+}\left(\dot{D}_{\mathfrak{I}}\right)
\end{aligned}
$$

where $w_{\mathfrak{I}}$ is surface Dupré energy, $k_{\mathfrak{I}}$ is a parameter measuring the nonlocal effect, $I_{-}$is the indicator function of the set $[-\infty, 0], \bullet$ indicates the scalar product, $\mathbf{N}$ is the outward versor normal to the domain $\Omega_{1}$ in correspondence of the interface $\mathfrak{I}, \hat{k}_{\mathfrak{I}}$ is the interface stiffness, $k_{\mathfrak{I}, \Omega}$ is the surface-domain interaction parameter and $c_{\mathfrak{I}}$ is the viscosity parameter of interface damage. Moreover, a further nonlocal interface effect is also introduced in the FF model. 
The derivatives of the free energies with respect to the observable and internal variables lead to the state equations. The derivative of the pseudo-potentials of dissipation with respect to the damage variable allows to derive the evolutionary law for the damage.

Balance equation are recovered in the FF model using the virtual power principle.

The following remarks regarding the FF model can be reported:

- both the continuum damage model and the interface damage model are nonlocal; in particular, the nonlocal effect is accounted for by the presence of the squares of the damages gradient appearing in the free energies $\Psi_{\Omega}$ and $\Psi_{\mathfrak{I}}$;

- damage and strain localizations are avoided by the nonlocal damage effect and by the presence of the viscosity in the damage evolution law;

$\circ$ the interface-body damage coupling is due to the presence of the term $k_{\mathfrak{I}, \Omega}\left(D_{\mathfrak{I}}-D_{\Omega}\right)^{2}$ in the definition of the free energy $\Psi_{\mathfrak{I}}$

o the interface damage and the body damage evaluated on the surface of adhesion are constrained, in the limit that $k_{\mathfrak{I}, \Omega} \rightarrow \infty$, to assume the same value;

- because of the damage coupling and of the nonlocal damage diffusion, if the body damage evolves, then the interface damage occurs; analogously, the growth of the interface damage induces the body damage evolution.

\section{THE NONLOCAL INTERFACE-BODY DAMAGE MODEL}

he mechanical system under investigation is composed by the body $\Omega_{2}$, whose behavior is considered linear elastic, in adhesion with the body $\Omega_{1}$, characterized by a quasi-brittle cohesive response, by means of a glue whose mechanical properties are much better that those of the support cohesive material. In such a case, the interface model describes the overall response of the glue material and of a thin layer on the surface of the support material. Experimental evidences show that the body damage strongly influences the degradation state of the interface, reducing the mechanical properties of the thin layer on the surface of the support material. On the contrary, the damage and also the failure of the interface does not induces damage diffusion inside the body $\Omega_{1}$. In other words, if the body $\Omega_{1}$ is damaged the degradation of the interface occurs; but it is possible to induce damage and even failure of the interface without damaging the body, i.e. the body $\Omega_{2}$ can be detached from the body $\Omega_{1}$ without inducing degradation of the mechanical properties of the body $\Omega_{1}$, unless of the thin layer of material which, indeed, is modeled by the interface. For this reason, the idea is to develop a interface-body damage model whose coupling is governed by a unilateral effect: $D_{\mathfrak{J}} \geq D_{\Omega}$.

A very simple body damage model is considered, developed in the two-dimensional framework. As a softening constitutive law is introduced for the body $\Omega_{1}$, localization of the strain and damage could occur. In order to overcome this pathological problem and, also, to avoid strong mesh sensitivity in finite element analyses, nonlocal constitutive law is considered. In particular, an integral nonlocal model is adopted; in fact, the nonlocal value of the strain tensor evaluated as:

$$
\overline{\mathbf{\varepsilon}}(\mathbf{x})=\frac{1}{\int_{\Omega_{1}} \psi(\mathbf{x}-\mathbf{y}) d \Omega} \int_{\Omega_{1}} \psi(\mathbf{x}-\mathbf{y}) \boldsymbol{\varepsilon}(\mathbf{y}) d \Omega
$$

where $\psi(\mathbf{x})$ is a weight function. It is assumed that the degradation of the cohesive material is due to the positive principal strain $[11,12]$; thus, the equivalent strain is introduced:

$$
\varepsilon_{e q}=\sqrt{\left\langle\bar{\varepsilon}_{1}\right\rangle^{2}+\left\langle\bar{\varepsilon}_{2}\right\rangle^{2}}
$$

where $\bar{\varepsilon}_{1}$ and $\bar{\varepsilon}_{2}$ are the nonlocal principal strains and the symbol exponential damage evolution law is considered:

$$
D_{\Omega}=\max _{\text {history }}\left\{\min \left\{1, \tilde{D}_{\Omega}\right\}\right\} \quad \tilde{D}_{\Omega}=\frac{\varepsilon_{e q}-\varepsilon_{0} e^{-k\left(\varepsilon_{e q}-\varepsilon_{0}\right)}}{\varepsilon_{e q}}
$$


where the starting value of $D_{\Omega}$ is zero, $\varepsilon_{0}$ is the initial threshold damage strain and $k$ is a material parameter associated to the damage energy.

About the evolution of the interface damage parameter $D_{\mathfrak{I}}$, a model which accounts for the coupling of mode I of mode II of fracture is considered [13,14]. In fact, the two quantities $\eta_{N}$ and $\eta_{T}$, defined as the ratios between the first cracking relative displacement $s_{N}^{0}$ and $s_{T}^{0}$ and the full damage relative displacement $s_{N}^{f}$ and $s_{T}^{f}$ are introduced:

$$
\eta_{N}=\frac{s_{N}^{0}}{s_{N}^{f}}=\frac{s_{N}^{0} \tau_{N}^{0}}{2 G_{c N}}, \quad \eta_{T}=\frac{s_{T}^{0}}{s_{T}^{f}}=\frac{s_{T}^{0} \tau_{T}^{0}}{2 G_{c T}}
$$

where $\tau_{N}^{0}$ and $\tau_{T}^{0}$ are the peak stresses on the first cracking relative displacement and $G_{c N}$ and $G_{c T}$ are the specific fracture energies in mode I and mode II, respectively. Then, the parameter $\eta$, which relates the two modes of fracture, is defined as follows:

$$
\eta=\frac{\left\langle s_{N}\right\rangle_{+}^{2}}{\|\mathbf{s}\|^{2}} \eta_{N}+\frac{s_{T}{ }^{2}}{\|\mathbf{s}\|^{2}} \eta_{T}
$$

Then, the relative displacement ratios are introduced:

$$
Y_{N}=\frac{\left\langle s_{N}\right\rangle}{s_{N}^{0}} \quad Y_{T}=\frac{s_{T}}{s_{T}^{0}}
$$

and the equivalent relative displacement ratio is considered:

$$
Y=\sqrt{Y_{N}^{2}+Y_{T}^{2}}
$$

Finally, the damage parameter is assumed to be a function of the history of relative displacement as follows:

$$
D_{\mathfrak{I}}=\max _{\text {history }}\left\{\min \left\{1, \tilde{D}_{\mathfrak{I}}\right\}\right\}
$$

where the parameter $\tilde{D}_{\mathfrak{J}}$ can be expressed by the relationship :

$$
\tilde{D}_{\mathfrak{\Im}}=\frac{Y-1}{Y(1-\eta)}
$$

Note that the Eq. (17) allows to obtain a linear stress - relative displacement relationship when pure mode I or pure mode II is activated. In fact, setting for instance $s_{T}=0$, formula (17) becomes:

$$
\tilde{D}_{\Im}=\frac{Y_{N}-1}{Y_{N}\left(1-\eta_{N}\right)}=\frac{s_{N}-s_{N}^{0}}{s_{N}\left(1-\eta_{N}\right)}
$$

Thus, in the softening phase, the normal stress at the interface results:

$$
\tau_{N}=\left(1-\tilde{D}_{\mathfrak{I}}\right) K_{N} s_{N}=\left(s_{N}^{f}-s_{N}\right) \frac{K_{N}}{s_{N}^{f}-s_{N}^{0}} s_{N}^{0}
$$

which is a linear relation between the stress $\tau_{N}$ and the relative displacement $s_{N}$. Analogously, setting $s_{N}=0$, the tangential stress is related to $s_{T}$ by the linear relationship:

$$
\tau_{T}=\left(1-\tilde{D}_{\mathfrak{J}}\right) K_{T} s_{T}=\left(s_{T}^{f}-s_{T}\right) \frac{K_{T}}{s_{T}^{f}-s_{T}^{0}} s_{T}^{0}
$$

Indeed, as previously emphasized, the interface damage depends also on the state of deterioration of the support material, i.e. on the damage occurring in the body $\Omega_{1}$. Thus, the body damage $D_{\Omega}$ has to be evaluated on the surface corresponding to the interface $\mathfrak{I}$. Once the interface damage $D_{\mathfrak{I}}\left(\mathbf{x}^{\mathfrak{I}}\right)$ and the body damage $D_{\Omega}\left(\mathbf{x}^{\mathfrak{I}}\right)$, are evaluated, the damage state of the interface $D_{\mathfrak{I}}^{c}\left(\mathbf{x}^{\mathfrak{I}}\right)$ is set as the maximum between the two obtained values, i.e.: 


$$
D_{\mathfrak{I}}^{c}\left(\mathbf{x}^{\mathfrak{I}}\right)=\max \left\{D_{\mathfrak{I}}\left(\mathbf{x}^{\mathfrak{I}}\right), D_{\Omega}\left(\mathbf{x}^{\mathfrak{I}}\right)\right\}
$$

where the superscript $c$ indicates that the evaluated damage accounts for the coupling between the interface and the body damages.

The following remarks can be emphasized:

o if the body $\Omega_{1}$ is damaged, implicitly the interface is even damaged; in other words, the damage state of the interface depends on the state of degradation of the body.

○ if the body $\Omega_{1}$ is not damaged, the interface damage is governed by the variable $D_{\Im}$, i.e. on the relative displacement occurring on the interface.

In order to perform a first and simple assessment of the proposed model, the problem proposed in previous section, as a possible delamination test, is considered again, but accounting for the coupling damage given in Eq. (21). The damage of the body indeed is equivalent to the reduction of the initial properties of the interface.

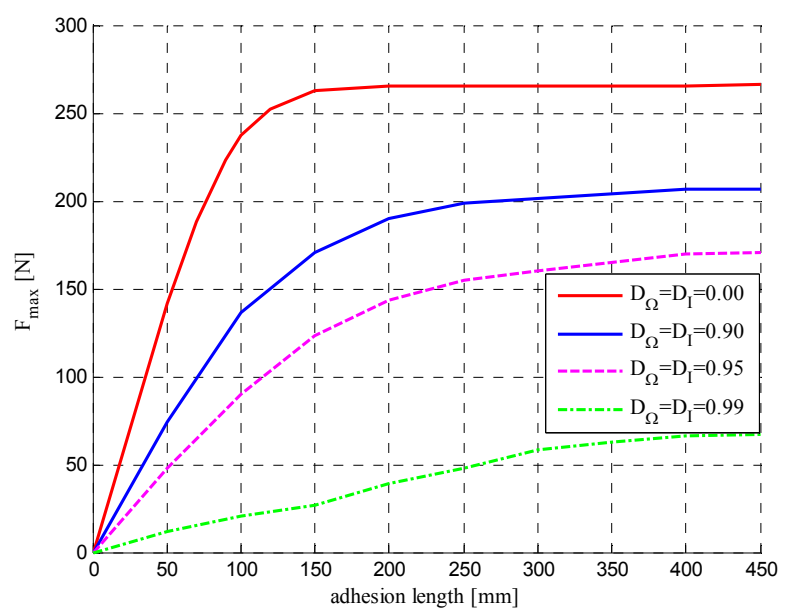

Figure 4: Decohesion force $F_{\max }$ versus adhesion length, coupled damage model.

In Fig. 4, the value of $F_{\max }$ is plotted versus the adhesion length. When the coupling between the interface and body damage is accounted for, the following observations can be remarked:

$\circ \quad$ as in the case of the uncoupled model, increasing the adhesion length $\ell$ the value of $F_{\max }$ grows, until the optimal adhesion length $\ell_{e}$ is reached, after which $F_{\max }$ remains constant;

- as in the case of the uncoupled model, for higher values of the damage state of the body $\Omega_{1}$ the optimal adhesion length increases;

- for higher values of the damage state of the body $\Omega_{1}$ the maximum value of $F_{\max }$ decreases.

This last results appears much more reliable with respect to the one obtained adopting the uncoupled damage model.

\section{CONCLUSIONS}

$\mathrm{I}$ $\mathrm{n}$ conclusion, a coupled interface-body nonlocal damage model is proposed. The presented application consider a very simple structural scheme, subjected to elementary loading and damage histories. Although the simplicity of the scheme and the damage evolution of the considered application, it is remarked the necessity of the use of the coupled damage model for the analysis of the reinforced concrete or masonry structural elements.

The research will continue implementing the proposed model in a finite element code in order to develop an effective tool for the strengthening design and to investigate on mode complex structural applications. 


\section{REFERENCES}

[1] T. C. Triantafillou, M. N. Fardis, Materials and Structures, 30 (1997) 486.

[2] T. C. Triantafillou, Journal of composites for constructions ASCE, 2 (1998) 96.

[3] M. R. Valluzzi, M. Valdemarca, C. Modena, Journal of composites for constructions ASCE, (2001) 163.

[4] S. Marfia, E. Sacco, International Journal of Solids and Structures, 38 (2001) 4177.

[5] J. F. Chen, Advances in Structural Engineering, 5 (2002) 37.

[6] P. Foraboschi, Journal of composites for constructions ASCE, (2004) 191.

[7] S. Marfia, M. Ricamato, E. Sacco, International Journal for Computational Methods in Engineering Science and Mechanics, 9 (2008) 77.

[8] E. Grande, G. Milani, E. Sacco, Engineering Structures, 30 (2008) 1842.

[9] M. A. Aiello, M. S. Sciolti, International Journal for Restoration of Buildings and Monuments, 9(6) (2003) 639.

[10] F. Freddi, M. Frémond, Journal of mechanics of materials and structures, 1(7) (2006).

[11] J. Mazars, Journal of Engineering Fracture Mechanics, 25 (1986) 729.

[12] J. Mazars, G. Pijaudier-Cabot, ASCE Journal of Engineering Mechanics, 115 (1989) 345.

[13] E. Sacco, J. Toti, Frattura ed Integrità Strutturale, 8 (2009) 3; DOI: 10.3221/IGF-ESIS.08.01.

[14] G. Alfano, E. Sacco, International Journal For Numerical Methods in Engineering, 68 (2006) 542. 